\title{
La innovación en el aula universitaria a través de la realidad aumentada. Análisis desde la perspectiva del estudiantado español y latinoamericano
}

\author{
Innovation in the University Classroom Through Augmented Reality. Analysis From the \\ Perspective of the Spanish and Latin American Students
}

\section{Inovação na sala de aula universitária através da realidade aumentada. Análise a parti da perspectiva de estudantes de espanhol e latino-americano}

Julio Cabero-Almenara

Universidad de Sevilla

Sevilla, España

cabero@us.es

https://orcid.org/0000-0002-1133-6031

Esteban Vázquez-Cano

Universidad Nacional de Educación a Distancia

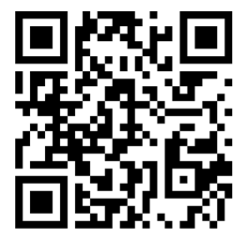

Madrid, España

evazquez@edu.uned.es

https://orcid.org/0000-0002-6694-7948

Wellington Remigio Villota-Oyarvide Universidad Católica Santiago de Guayaquil

Santiago de Guayaquil, Ecuador wellington.villota@cu.ucsg.edu.ec

https://orcid.org/0000-0002-0081-4704

Eloy López-Meneses Universidad Pablo de Olavide

Sevilla, España

elopmen@upo.es

https://orcid.org/0000-0003-0741-5367

Recibido • Received • Recebido: 18 / 06 / 2020

Corregido • Revised • Revisado: 14 / 04 / 2021

Aceptado•Accepted • Aprovado: 26 / 05 / 2021

\section{Resumen}

Objetivo. Este artículo analiza las experiencias de innovación universitaria con tecnologías inmersivas (realidad aumentada, RA) de diversas titulaciones de la Universidad Pablo de Olavide de Sevilla (España) y de la Universidad Católica de Santiago de Guayaquil (Ecuador) correspondiente al curso académico 2018-2019. Metodología. A través de una metodología mixta de análisis de datos 
http://doi.org/10.15359/ree.25-3.1

http://www.una.ac.cr/educare

educare@una.ac.cr

(cuantitativa y cualitativa) y el diseño de un cuestionario ad hoc de elaboración propia se analizaron las percepciones de 202 estudiantes en relación con las ventajas y desventajas de la RA y las apps de realidad aumentada utilizadas en los seminarios de Tecnologías Emergentes en contextos educativos, impartidos en ambas universidades. Resultados. Los resultados muestran que las aplicaciones móviles más valoradas fueron: QuiverVisión y Hp Revail por su interactividad, entorno usable y su proyección didáctica en contextos formativos, especialmente en edades tempranas. Conclusiones. El estudiantado percibe, como principales ventajas de la RA, el desarrollo de habilidades cognitivas y competenciales y, como principales desventajas, la necesidad de formación del profesorado universitario para la implementación en las aulas y los posibles efectos adversos de la brecha digital.

Palabras claves: Tecnología emergente; realidad aumentada; apps educativas; innovación universitaria.

\begin{abstract}
Objective. This article analyzes the experiences of university innovation with immersive technologies (Augmented Reality, RA) of various degree programs at the Pablo de Olavide University of Seville (Spain) and the Catholic University of Santiago de Guayaquil (Ecuador) corresponding to the academic year 2018-2019. Method. Through a mixed methodology of data analysis (quantitative and qualitative) and the design of an ad hoc questionnaire of own elaboration, the perceptions of 202 students were analyzed in relation to the advantages and disadvantages of the RA and the Augmented Reality apps used in the Emerging Technologies seminars in educational contexts, taught in both universities. Results. The results show that the most valued mobile applications were QuiverVisión and HP Reveal because of their interactivity, wearable environment, and educational projection in formative contexts, especially at early ages. Conclusions. Students perceive the development of cognitive skills and the development of competencies as the main advantages of RA. Likewise, as main disadvantages, the need for university teacher training for classroom implementation and the possible adverse effects of the digital divide.
\end{abstract}

Keywords: Technological education; augmented reality; educational apps; university innovation.

\title{
Resumo
}

Objetivo. Este artigo analisa as experiências de inovação universitária com tecnologias imersivas (Realidade Aumentada, RA) de vários graus da Universidade Pablo de Olavide de Sevilha (Espanha) e da Universidade Católica de Santiago de Guayaquil (Equador) correspondentes ao ano letivo de 2018-2019. Metodologia. Por meio de uma metodologia mista de análise de dados (quantitativa e qualitativa) e do desenho de um questionário ad hoc de elaboração própria, as percepções de 202 estudantes foram analisadas em relação às vantagens e desvantagens dos aplicativos RA e das aplicações de Realidade Aumentada utilizadas nos seminários de Tecnologias Emergentes em contextos educacionais, ministrados em ambas as universidades. Resultados. Os resultados mostram que as aplicações tecnológicas mais valorizadas foram: QuiverVisión e Hp Revail por sua interatividade, ambiente amigável e sua projeção educacional em contextos formativos, especialmente em idades precoces. Conclusão. É perceptível por parte de estudantes que, como principais vantagens da $A R$, seria o desenvolvimento de habilidades cognitivas e de competência e, como principais desvantagens, a necessidade de formação do corpo docente universitário para a implementação em sala de aula e os possíveis efeitos adversos da exclusão digital.

Palavras-chave: Tecnologia emergente; realidade aumentada; aplicativos educacionais; inovação universitária. 
http://doi.org/10.15359/ree.25-3.1

http://www.una.ac.cr/educare educare@una.ac.cr

\section{Introducción}

Nos encontramos en la actualidad ante el reto de innovar en todos los ámbitos y etapas educativas, pero más aún, si cabe, en los procesos de enseñanza-aprendizaje en la educación superior. Venimos de un siglo XX en el que la enseñanza transmisiva por medio del personal docente y un papel pasivo del estudiantado era el enfoque didáctico más común en las aulas universitarias. Con el advenimiento de la tecnología, el enfoque competencial del conocimiento en el que el estudiantado debe saber hacer más que saber teoría y la democratización en el acceso a los dispositivos digitales, el contexto didáctico de la educación superior está virando hacia enfoques didácticos más participativos en los que las tecnologías emergentes están resultando de gran ayuda (Brinson, 2017; Chang, 2018; Chao et al., 2016).

En este contexto, la realidad aumentada puede definirse como un sistema con las siguientes características: combina lo real con lo virtual, es interactivo en tiempo real y se desarrolla en 3D (Azuma, 1997). Esto nos permite interrelacionar lo digital con lo físico de forma dinámica a través de dispositivos digitales y operar con otros periféricos como gafas de realidad aumentada que habilitan un nuevo contexto de interacción y conocimiento de realidades a las que anteriormente solo se tenía acceso en dos dimensiones.

Tomando en consideración las premisas anteriores, nuevos contextos didácticos y tecnologías emergentes, este artículo tiene como principales objetivos presentar y describir varias experiencias didácticas en dos universidades, Universidad Pablo de Olavide de Sevilla (España) y Universidad Católica de Santiago de Guayaquil (Ecuador), y analizar la potencialidad didáctica de la realidad aumentada, sus posibles ventajas y desventajas desde la percepción del estudiantado universitario participante.

\section{La realidad aumentada en el ámbito educativo}

Hoy en día estamos inmersos en un cambio tecnológico perenne que ofrece nuevos retos a la sociedad, en general y a los procesos educativos, en particular (Froehlich, 2018; RodríguezGarcía et al., 2018). Asimismo, su uso pedagógico es un elemento prioritario en la actual educación (Jiménez Hernández et al., 2019), y su aplicabilidad didáctica en los contextos formativos resulta más beneficiosa que la metodología tradicional sustentada en la transmisión de contenidos y, a su vez, implica un cambio didáctico que favorece procesos de aprendizaje más dinámicos y significativos (Cabero-Almenara et al., 2018; López Belmonte et al., 2019). Además, como apunta Hood Cattaneo (2017), la RA está siendo incorporada de manera progresiva como una de las tecnologías emergentes con más proyección de futuro en el ámbito educativo, unida a pedagogías activas. En este sentido, la RA es una tendencia tecnológica de los últimos años que está teniendo hondas repercusiones en diferentes ámbitos sociales, científicos y educativos. En el ámbito educativo, sus aplicaciones permiten, al estudiantado, acercarse y conocer los sistemas más tradicionales desde enfoques más dinámicos y realistas (Fombona Cadavieco y Vázquez-Cano, 2017). Asimismo, la RA es accesible en apps instalables en teléfonos inteligentes y 
http://doi.org/10.15359/ree.25-3.1

http://www.una.ac.cr/educare

educare@una.ac.cr

tabletas, lo que facilita que el estudiantado sea autónomo en su utilización y que el profesorado en las aulas universitarias también pueda hacer un uso académico de los dispositivos que sus estudiantes traen a las clases.

Esta tecnología aumentada permite, como expresan diferentes textos (Cabero Almenara et al., 2020), crear nuevos entornos inmersivos con capas de realidad virtual que posicionan al estudiantado ante la simulación de situaciones y contextos que solo serían posibles en situaciones reales. Por ejemplo, cada estudiante puede ver en tres dimensiones los órganos interiores del cuerpo humano y entender, de una manera mucho más visual, el proceso de la alimentación. También, se pueden simular contextos, como la compra de un billete de avión y cómo se realizaría de forma virtual en una segunda lengua. Esta característica ayuda a que el alumnado adquiera más fácilmente una serie de competencias que van a facilitarle la adquisición del conocimiento que se le está requiriendo (Fombona Cadavieco y Vázquez-Cano, 2017).

Por otra parte, diferentes aestudios (Blas Padilla et al., 2019) indican que en el ámbito educativo la RA es uno de los avances tecnológicos transformadores de gran impacto, al permitir la creación de contenidos interactivos que se pueden visualizar al estudiantado e incorporar nueva información educativa en documentos impresos. De igual manera, su uso en el contexto educativo (Barroso Osuna y Gallego Pérez, 2017; Chang y Hwang, 2018; Han et al., 2015; Pedraza Goyeneche et al., 2017; Santos et al., 2016) podría ofrecer interesantes posibilidades, tales como: añadir información relevante y significativa al mundo real para que sea más inteligible al estudiantado; promover el aprendizaje ubicuo; la creación de contextos virtuales simulados (por ejemplo, laboratorios) más seguros para el alumnado, favorecer al estudiantado para que sea diseñador y evaluador de materiales educativos con tecnología RA y utilizar e-actividades de capacitación basadas en la metodología Flipped Classroom y promover el aprendizaje informal.

Según, Fernández Robles (2018), la RA permite hacer efectivas diferentes competencias genéricas y transversales en elámbito universitario; derivado, principalmente, de su potencialidad en la integración de metodologías activas que permiten que el estudiantado participe, de una forma más situada, y entienda procesos complejos desde las posibilidades inmersivas de este tipo de tecnología. Además, de todo lo expuesto con anterioridad, la utilización de objetos basados en la tecnología aumentada presenta altos indicadores de motivación y satisfacción en el estudiantado y mejora los resultados de aprendizaje (Chang et al., 2013; Han et al., 2015; Wojciechowski y Cellary, 2013), a su vez, en este ámbito adquiere mayor relevancia, debido a su facilidad de uso y a la disponibilidad de los dispositivos técnicos para el estudiantado (Cabero Almenara et al., 2020).

Asimismo, la utilización de este tipo de tecnología produce un aumento significativo de los niveles de motivación (Wojciechowski y Cellary, 2013), aunque, también, la realidad aumentada tiene una serie de obstáculos y dificultades que no se deben obviar: como la posible brecha digital por falta de recursos económicos para su implementación y también puede provocar ciertas desigualdades en colectivos sociales vulnerables por falta de formación. Además, se debe progresar 
http://doi.org/10.15359/ree.25-3.1

en la accesibilidad y usabilidad de objetos de aprendizaje aumentados (Cabero-Almenara et al., 2018). No obstante su integración curricular, en los contextos universitarios es fácil, pues la mayoría del alumnado dispone de móviles (Fombona Cadavieco y Vázquez-Cano, 2017).

El empleo de metodologías emergentes como la realidad aumentada permite afrontar el proceso de enseñanza-aprendizaje desde una dimensión más innovadora al permitir la creación de contenido por parte del estudiantado. Esta actividad de creación entronca con una necesidad de desarrollo de las competencias genéricas y transversales de estudiantes. Cuando el estudiantado recibe contenido que debe crear y (co)crear se fomenta el desarrollo de estrategias de necesarias para la mejora de sus competencias personales, académicas y profesionales, ya que pone en práctica habilidades para buscar, obtener, procesar y comunicar y, por último, transformar en conocimiento (Vázquez-Cano, 2021). Estos enfoques didácticos tienen su repercusión en los modelos de aprendizaje de estudiantes e incrementan su valor cuando se añade el componente dialógico y el trabajo colaborativo, por medio del conectivismo. El objetivo es proporcionar a las tareas educativas un contexto auténtico en el que el material debe favorecer tanto la adquisición de contenidos como un proceso de creación de este mismo y, de esta manera, fomentar el desarrollo de las competencias transversales y genéricas necesarias al finalizar los estudios universitarios (Cozar et al., 2015). La RA puede ayudar a incrementar la motivación del estudiantado y favorecer mayores cuotas de creatividad. En este sentido, existe ya un amplio número de investigaciones que evidencia sus beneficios con respecto al aprendizaje por descubrimiento (Han et al., 2015; Kim et al., 2016).

Por último, esta tecnología emergente "puede implicar un gran salto cualitativo en la enseñanza, ya que transforma la percepción de la realidad física de los estudiantes para que puedan tomar el control de su propio aprendizaje" (Blas Padilla et al, 2019, p. 38). A su vez, "esta tecnología [inmersiva] está creciendo en los últimos años gracias a los dispositivos digitales móviles que facilita su acceso a todo el público (Aznar-Díaz et al., 2018, citados en Blas Padilla et al., 2019, p. 38).

\section{Metodología}

Las diversas experiencias aquí presentadas se desarrollaron a través de seminarios didácticos de formación en realidad aumentada, en concreto, en la Universidad Pablo de Olavide (UPO), durante los meses de febrero y marzo del curso académico 2018-19 en dos asignaturas: Tecnologías de la Información y la Comunicación en Educación Social, correspondientes ambas al primer año de la titulación del Grado de Educación Social y Doble Grado de Educación Social y Trabajo Social de la Facultad de Ciencias Sociales de la Universidad Pablo de Olavide, de Sevilla (España) con una carga de 7,3 créditos ECTS (European Credit Transfer System) y durante los meses de enero y febrero y en la Universidad Católica de Santiago de Guayaquil (UCSG), en la titulación de Ingeniería en Dirección y producción en Artes Multimedia durante los meses de mayo y junio del mismo curso académico. 
http://doi.org/10.15359/ree.25-3.1

http://www.una.ac.cr/educare

educare@una.ac.cr

Por otra parte, los seminarios inmersivos de ambas instituciones de educación superior pretendían alcanzar los siguientes objetivos educativos: (1) Estudiar nuevas aplicaciones móviles relacionadas con la realidad aumentada para su aplicación en ámbitos formativos. (2) Potenciar en el estudiantado actitudes proactivas para el uso de los recursos tecnológicos aumentados en contextos educativos. (3) Favorecer la alfabetización digital vinculadas a la utilización de las apps presentadas en los seminarios educativos de realidad aumentada (RA).

Los seminarios de innovación en tecnología emergente se impartían al alumnado de ambas universidades con una duración de tres sesiones de dos horas y consistía en una breve explicación teórica sobre tecnología aumentada y sus niveles, además de sus fortalezas y debilidades en el ámbito formativo. Además del uso práctico de diversas aplicaciones de RA en contextos educativos (Quiver, Chromville, ArFlashcards y Hp Revail). Para ello, se utilizaban en las aulas universitarias diferentes dispositivos móviles para el funcionamiento por georreferenciación a través de la visualización de marcadores o símbolos en papel (Marker Traicking). Las app de RA utilizadas en las sesiones prácticas fueron las siguientes: (1) QuiverVision: es una apps aumentada con plantilla en papel para dibujarlas. Su URL es: http://quivervision.com y luego al visualizarla con el móvil a través de su cámara, dichas láminas coloreadas adquieren vida generando escenarios aumentativos para mejorar los procesos de aprendizaje. La aplicación permite interactuar con el modelo creado y posicionar al estudiante ante una situación real. Es accesible desde iOS y Andorid. (2) Chromville: es una aplicación similar a la app de Quiver sustentada en láminas para colorear que ayuda a la comprensión didáctica de conceptos. Es una aplicación que pretende fusionar la tecnología para promover la creatividad e integrar las ocho inteligencias múltiples. Para ello, incluye una serie de láminas para colorear para acercar la ciencia al estudiantado a través de varios módulos temáticos. Ha sido elegida como una de las 40 app por la empresa Apple como una aplicación puntera en educación. Su enlace es https://chromville.com (3) Ar Flascards. Esta app educativa de lecto-escritura permite conocer y experimentar a través de fichas de realidad aumentada el abecedario completo en inglés que al visualizarlo a través de la app aparecerá un animal vinculado con la letra del marcador. Permite también crear tarjetas interactivas digitales que diferentes animales en pantalla en 3D, y al tocar el animal se puede escuchar su nombre. Su URL es: http://arflashcards.com/getflashcards/ (4) HP Reveal (LinkReader). Es una aplicación de realidad aumentada multiplataforma (antiguamente conocida como Aurasma) que permite la elaboración de contenidos formativos y fichas educativas que utilizan auras (unión de un marcador con su capa de información). Se encuentra disponible en AppStore y GooglePlay. Más información acerca de esta aplicación: https://studio.hpreveal.com/landing. A su vez, se elaboró un espacio virtual (Figura 2), que ofrecía una recopilación de otras apps de carácter educativo, tutoriales y noticias de interés (http://cort.as/x-F4) para ambas universidades. 
http://doi.org/10.15359/ree.25-3.1

Al finalizar el seminario formativo se solicitaba a cada estudiante cumplimentar un cuestionario elaborado ad hoc titulado: Uso didáctico de la RA, donde uno de sus ítems es indagar qué app de RA utilizadas en dicho seminario formativo de RA era la más motivadora y relevante para los ámbitos educativos. Su enlace es: https://goo.gl/forms/STik3sl9KdPzZi773. Asimismo, cada estudiante de la Universidad Pablo de Olavide también tenía que desarrollar un edublog personal para la asignatura sobre las evidencias de las actividades realizadas en el segundo semestre académico. En última instancia, cabe mencionar que más de la mitad $(61,88 \%)$ del estudiantado de ambas universidades que participaron en los seminarios indicaron en el cuestionario aplicado que no tenían conocimientos sobre esta tecnología inmersiva.

La metodología de la investigación fue de corte mixto: cuantitativo, cualitativo y descriptivo. Se aborda un estudio exploratorio cuyo principal objetivo es conocer las apps educativas de RA más motivadoras y educativas empleadas en los seminarios formativos de RA durante el curso académico 2018-19 en la UPO y UCSG. Para tal menester, se parte de enfoque metodológico con base en la etnografía virtual que permita analizar la interacción y participación del estudiantado en los entornos virtuales, a la vez que se permite su propia autorreflexión; una dinámica que ayuda a formación en competencias genéricas y transversales en el Espacio Europeo de Educación Superior (Pool-Cibrian y Martínez-Guerrero, 2013).

El análisis cualitativo consistió en diversas etapas, la primera de carácter analítico consistente en el análisis descriptivo de las unidades semánticas más relevantes y su categorización grupal. Posteriormente, se efectuó su codificación e inferencia interpretativa en la generación y recopilación sistémica de metacategorías funcionales.

La muestra participante estuvo compuesta por 202 estudiantes de la Universidad Pablo de Olavide y de la Universidad Católica Santiago de Guayaquil con las siguientes características: 59 estudiantes ( 6 hombres y 53 mujeres), correspondientes a la titulación de Grado en Educación Social; 60 estudiantes (4 hombres y 56 mujeres), pertenecientes a la titulación de Doble Grado en Trabajo Social y Educación Social, ambas titulaciones vinculadas a la Universidad Pablo de Olavide, Sevilla (España) y 83 estudiantes (43 hombres y 40 mujeres) procedentes de la Universidad Católica de Santiago de Guayaquil (Ecuador).

Al terminar los seminarios de formación en RA, el estudiantado rellenaba un cuestionario implementado en google forms. Su enlace es: https://tinyurl.com/y3fuzrl6. En este sentido, se tuvo para su diseño el marco de referencia realizado por Barroso Osuna y Gallego Pérez (2017) y se validó por el método Delphi en doble ronda. Los datos fueron analizados con SPSS. Para determinar la fiabilidad del cuestionario se calculó el coeficiente alpha de Cronbach para las puntuaciones de la valoración. Los valores del coeficiente alpha de Cronbach fueron superiores a 0,7, por lo que podemos afirmar que el cuestionario tenía alta fiabilidad. Para comprobar la validez del cuestionario se aplicó un análisis factorial a las valoraciones. La medida de Kaiser-Meyer-Olkin fue superior a 0,75. Además, también se rechazó la hipótesis nula de la prueba de esfericidad de Barlett. 
http://doi.org/10.15359/ree.25-3.1

http://www.una.ac.cr/educare

educare@una.ac.cr

Unos de los objetivos del cuestionario era recabar las percepciones del estudiantado en relación con las diversas apps de RA más motivadoras y educativas usadas en el seminario de innovación tecnológica. En concreto, en nuestro estudio se analizaron las apreciaciones e impresiones de los 202 estudiantes de las universidades UPO y UCSG. Por último, se utilizaban durante las sesiones prácticas estrategias metodológicas basadas en clicker (sistemas de respuesta de la audiencia), es decir, herramientas que se pueden aplicar en el contexto educativo como feed-back con el alumnado, muy útil para monitorizar su aprendizaje (Caldwell, 2007). En nuestro caso, se utilizaba para indagar sobre las apps más motivadoras del seminario y dinamizar debates colectivos sobre las tecnologías inmersivas. En concreto, las apps utilizadas en el seminario innovador fueron proyectadas donde todos los estudiantes y las estudiantes las visualizaban y contestaban mediante su teléfono móvil, portátil o tablet. En la Figura 1 se muestra un ejemplo realizado en una sesión práctica de un grupo de estudiantes de Doble Grado en Trabajo Social y Educación Social. Por último, en concordancia con Hunsu et al. (2016), estimamos que este tipo de metodología basada en clickers tiene efectos positivos sobre el aprendizaje cognitivo.

Figura 1: Ejemplificación de la valoración de un grupo de estudiantes de la
UPO en relación con diversas aplicaciones móviles de RA empleadas en el
Seminario de tecnologías emergentes a través de la aplicación Mentimeter

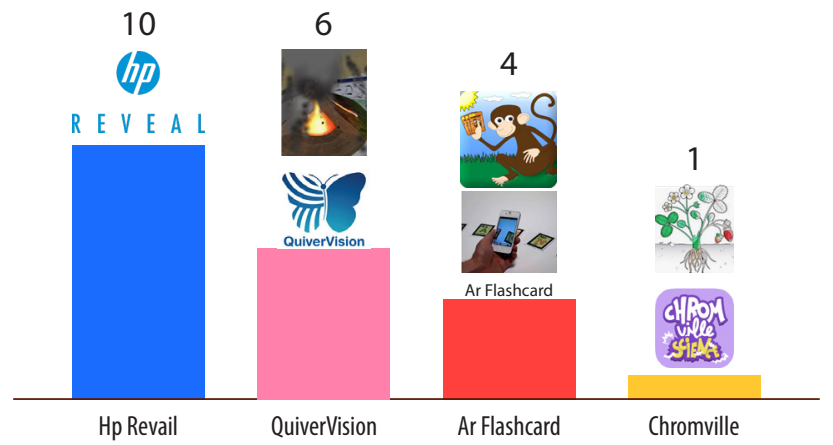

Nota: Elaboración propia.

\section{Resultados y discusión}

En este apartado se analizan las 202 percepciones del estudiantado de la Facultad de Ciencias Sociales de la UPO e Ingeniería en Producción y Dirección en Artes Multimedia de la UCSG, correspondientes al curso académico 2018/19 sobre las apps de RA más motivadoras y didácticas empleadas en las sesiones de los seminarios de realidad aumentad en contextos educativos. Para ello, en una primera instancia, se recopila, en la Figura 2, la frecuencia de respuesta del alumnado en relación con las aplicaciones de RA más significativas desde un punto de vista formativo y usable utilizadas en el seminario inmersivo de la titulación de Grado de Educación Social del curso académico 2018-19. A su vez, cabe mencionar que más del 80\% ( $\mathrm{f}=49$ ) del estudiantado de esta titulación ha seleccionado la app QuiverVision como la aplicación 
http://doi.org/10.15359/ree.25-3.1

móvil más interesante y la mayoría fundamenta su decisión por su dinamismo, interactividad, usabilidad y relevancia didáctica para utilizarse en contextos formativos.

Figura 2: Frecuencias de respuestas del estudiantado de Grado de Educación Social, relativas a las apps de RA más motivadoras utilizadas en el Seminario formativo (2018-19)

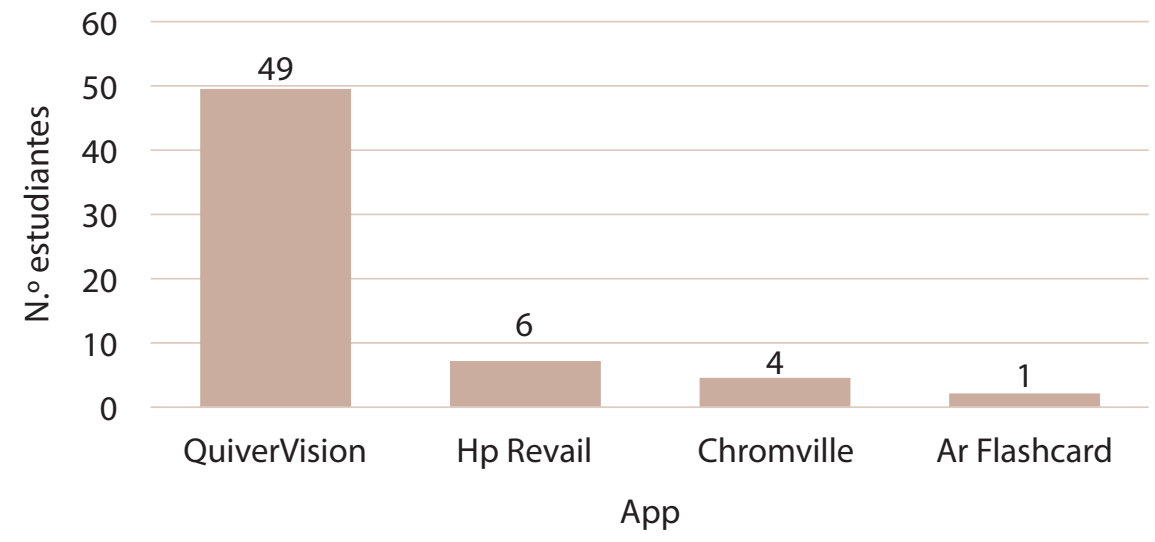

Nota: Elaboración propia.

En referencia al análisis porcentual del estudiantado de la siguiente titulación se obtuvo: Doble Grado en Trabajo Social y Educación Social, constituido por 60 estudiantes valoraron las apps de RA de forma similar a la anterior titulación, en concreto, las apps: QuiverVision (75\%); Hp Revail (8,33\%) y, en último lugar, la app AR Flashcard (1,67\%) era muy interesante para los procesos de aprendizaje en idiomas. En cambio, en esta titulación la app Chromville la estimaron de mayor valor didáctico con un 15\% (Figura 3).

Figura 3: Porcentaje de las app de RA utilizadas en el Seminario de Realidad Aumentada en contextos educativos de la titulación de Doble Grado en Trabajo Social y Educación Social (2018-19)

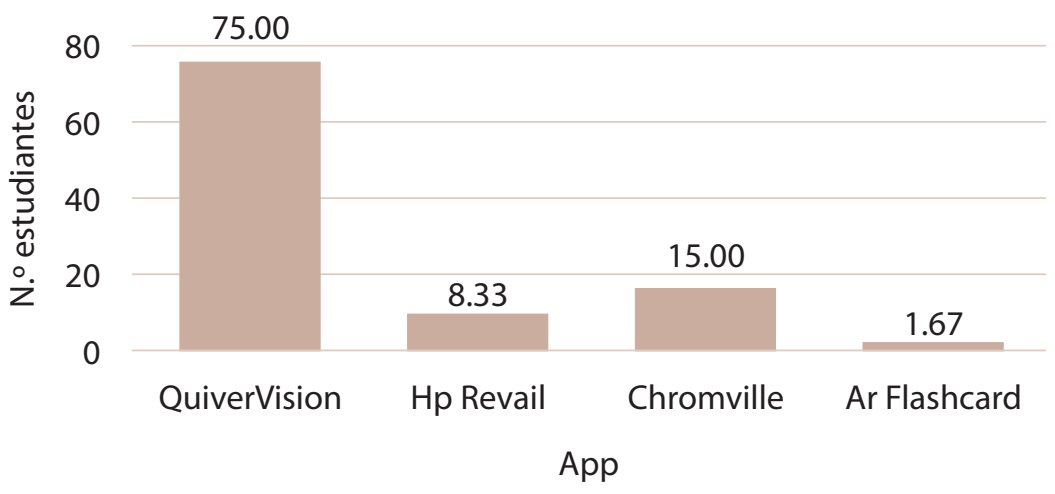

Nota: Elaboración propia. 
http://doi.org/10.15359/ree.25-3.1

http://www.una.ac.cr/educare

educare@una.ac.cr

En cuanto a su fundamentación teórica, expresaban, en la misma línea discursiva, que la anterior titulación, la app QuiverVision era la más interesante desde un punto de vista didáctico por ser muy intuitiva, fácil de manejar, permitir colorear las láminas y luego convertirlas en objetos animados y enriquecer los procesos de aprendizaje, espacialmente en edades tempranas. Seguidamente, se muestra en la Figura 4 la frecuencia de respuesta de las percepciones de los 83 estudiantes, correspondiente a la titulación de Ingeniería en Producción y Dirección en Artes Multimedia, correspondiente al curso académico 2018-19 procedentes de la Universidad Católica de Santiago de Guayaquil (Ecuador).

Figura 4: Frecuencias de respuestas del estudiantado de la Universidad Católica de Santiago de Guayaquil, Ecuador

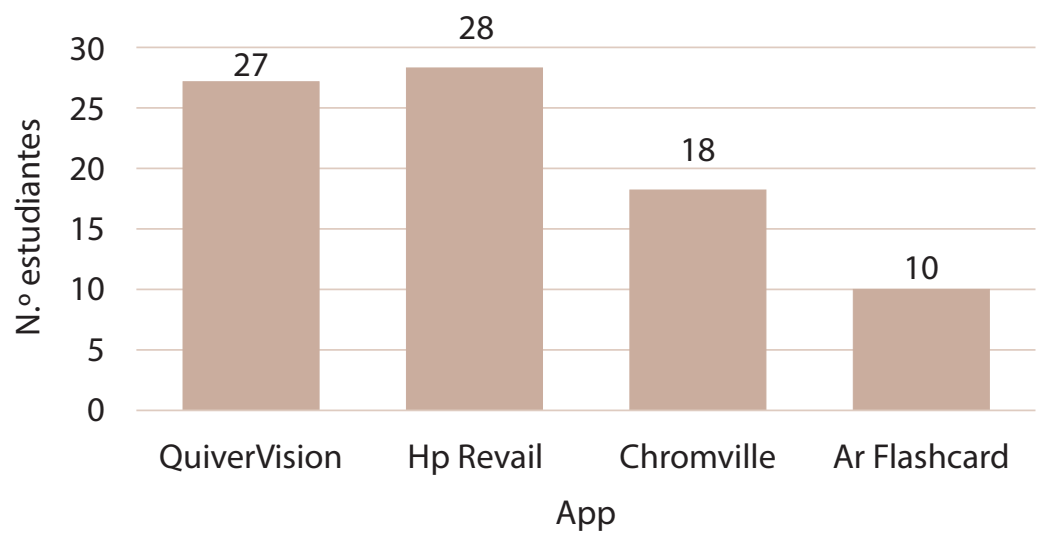

Nota: Elaboración propia.

En este sentido, como se infiere de la Figura 4, el estudiantado de la titulación de Ingeniería en Producción y Dirección en Arte Multimedia, opinaron que las app más relevantes utilizadas en el Seminario de Tecnología Emergente eran: HpRevail $(\mathrm{f}=28 ; 33,73 \%)$ y Quiversion ( $f=27 ; 32,53 \%$ ) por ser más realistas, fácil de manejar. "Además, de ofrecer escenarios formativos amigables para la comunicación, el fomento de la gamificación en escenarios formativos y ser recursos didácticos muy adecuados y funcionales para su futuro profesional en contextos sociales" (Blas Padilla et al, 2019, p. 44). Asimismo, la app HP Revail, como apuntan diferentes estudiantes, es una aplicación que se puede realizar con diferentes tipos de realidad aumentada tanto como con vídeos imágenes y en 3D, de una manera fácil, sin muchas complicaciones. Otra parte apunta que no hace falta una lámina para que funcione. $Y$ en referencia a las otras dos app, aparecen con un menor porcentaje Chromville (21,69\%) y Ar Flashcards (12,05\%) por ser más específicas y menos intuitivas. 
http://doi.org/10.15359/ree.25-3.1 http://www.una.ac.cr/educare educare@una.ac.cr

Por otra parte, se muestran en la Figura 5 los resultados de frecuencias de las tres titulaciones de ambas universidades "para observar su oscilación referente a las apps de RA más motivadoras y útiles para los procesos formativos utilizadas en el Seminario de tecnologías emergentes" (Blas Padilla et al, 2019, p. 45). En este sentido, el estudiantado que cursa en la UPO estima más relevante la app QuiverVision por su proyección didáctica en edades tempranas; el estudiantado de la USG considera la app Hp Revail por su facilidad de manejo y su aplicabilidad en escenarios formativos. Y en última instancia, consideran la app Ar Flashcard en las tres titulaciones de menor relevancia por ser una app más específica y orientada a un intervalo de edades más concreto.

Figura 5: Comparativa de las frecuencias de las tres titulaciones correspondientes al curso académico 2018-19

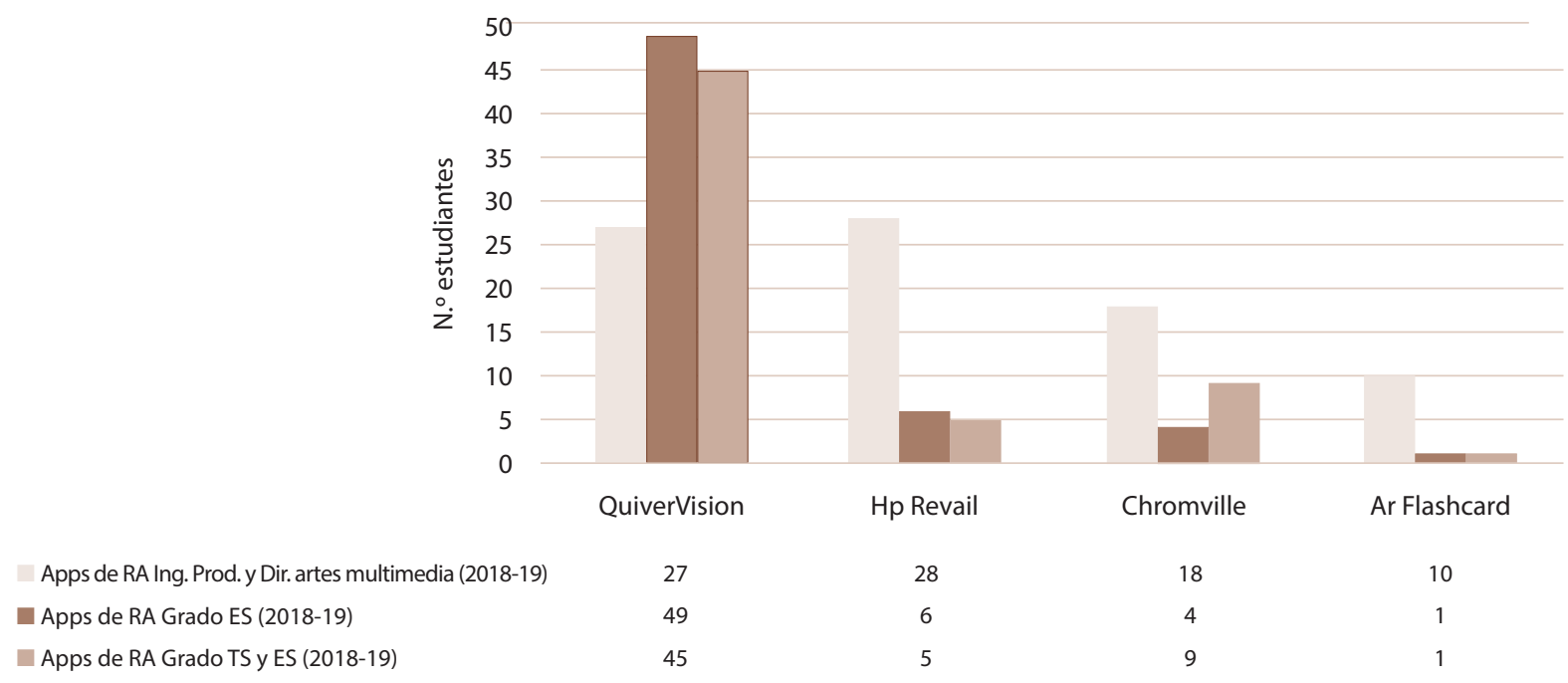

APP

Nota: Elaboración propia.

A continuación, en la Figura 6, presentamos la red de Atlas-Ti de ventajas y desventajas de la aplicación de la RA en las aulas universitarias desde la perspectiva del estudiantado universitario. 
http://doi.org/10.15359/ree.25-3.1

http://www.una.ac.cr/educare

educare@una.ac.cr

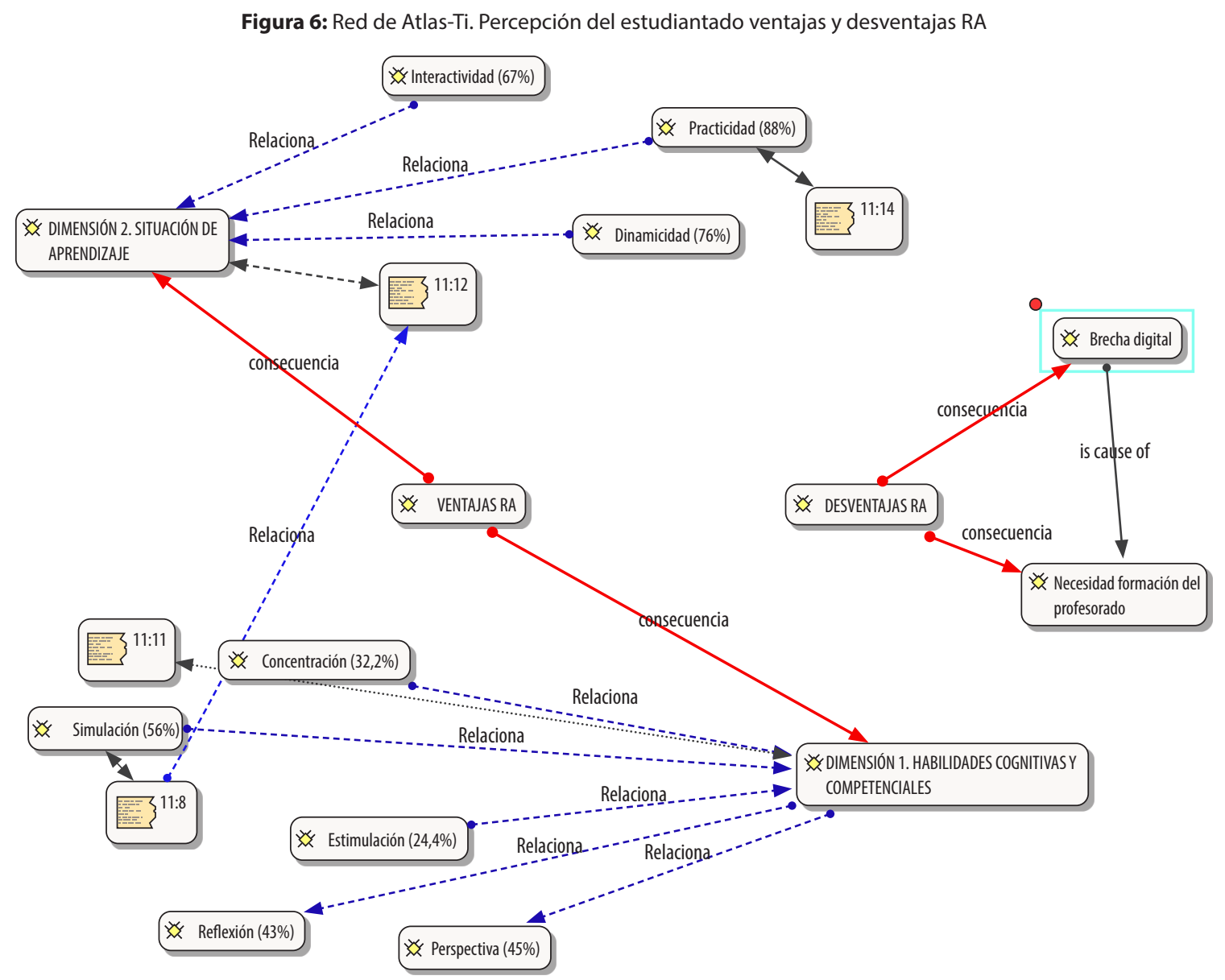

Nota: Elaboración propia.

Con respecto al análisis cualitativo de las ventajas y desventajas de la RA en el ámbito universitario, el estudiantado de ambas universidades considera que el uso didáctico de la RA tiene varias ventajas que se pueden agrupar en dos ámbitos. En mejora de las habilidades cognitivas y competenciales en los que se pueden agrupar las siguientes categorías: estimulación $(24,4 \%)$, concentración $(32,2 \%)$, simulación $(56 \%)$, reflexión (43\%) y perspectiva $(45 \%)$. Por otro lado, la situación de aprendizaje en la que se valora como principales ventajas: interactividad (67\%), practicidad (88\%) y dinamicidad (76\%). En este sentido, los resultados obtenidos se corroboran en otros estudios (Moreno Martínez y Leiva Olivencia, 2017). De igual manera, el empleo de objetos de aprendizaje aumentado en los ecosistemas bióticos de aprendizaje favorece la motivación, la atención y los procesos de aprendizaje interactivos (Cózar Gutiérrez et 
http://doi.org/10.15359/ree.25-3.1

al., 2015; Wojciechowski y Cellary, 2013). Estas ventajas han demostrado una mayor profundidad en las etapas de la educación primaria y secundaria, ya que la motivación de estudiantes y su vinculación con las competencias es mayor, a la vez que permite también salidas de campo virtuales con niñez de edades más tempranas (Cozar et al., 2015). En este sentido, la interactividad es otra de las ventajas que los estudios han remarcado con mayor intensidad (Billinghurst y Dunser, 2012; Bressler y Bodzin, 2013). Una interactividad que permite afrontar el proceso de enseñanza-aprendizaje desde el trabajo en grupo y cooperativo.

En relación con las posibles desventajas, el estudiantado universitario señala dos ámbitos de mejora: la brecha digital (56\%) y la necesidad de formación del profesorado (76\%). El estudiantado percibe que tecnologías emergentes como la RA posibilitan nuevas formas de aprender y desarrollar competencias, pero su adopción viene condicionada por la necesaria formación del profesorado universitario que, en la mayoría de las ocasiones, no se somete a procesos formativos para favorecer la innovación didáctica en las aulas de la educación superior. Esta situación puede agravarse y, unida a la falta de adopción de estas tecnologías, producir divergencias en la formación del estudiantado, pues este verá implementadas estas tecnologías solo por docentes que innoven y no como una didáctica habitual en las aulas, lo que puede conllevar una brecha digital en la formación del estudiantado universitario.

En línea con la formación del profesorado, una de las principales desventajas es la falta de adopción de un modelo de competencias docentes en tecnologías digitales que permita afrontar con solvencia. Para ello, es necesario potenciar la formación en consonancia con los descriptores internacionales que abordan las competencias en cinco áreas: (1) Información y alfabetización informacional. (2) Comunicación y colaboración. (3) Creación de contenidos digitales. (4) Seguridad. (5) Resolución de problemas (Carretero et al., 2017; Instituto Nacional de Tecnologías Educativas y de Formación del Profesorado [INTEF], 2017). Para una correcta integración adecuada y didáctica de la RA en la educación superior, se precisa implementar programas formativos en competencias clave como las siguientes: Evaluación de información, datos y contenidos; gestión de la identidad digital; integración y reelaboración de contenidos digitales, derechos de autoría y licencias; protección de datos personales e identidad digital e innovación y uso de la tecnología digital de forma creativa, entre otros.

Asimismo, es importante llamar la atención sobre posibles problemas que pueden surgir con el empleo de esta tecnología, como pueden ser: la brecha digital causada por problemas económicos o formativos. Hay que tener en cuenta que la correcta implementación de estas didácticas requiere de un proceso formativo que permita su correcta y adecuada aplicación en las diferentes etapas del sistema educativo. Asimismo, en ocasiones, puede generar un desembolso económico en dispositivos y aplicaciones que puede limitar su empleo y generar, así, una mayor brecha digital (Chiang et al., 2014). 
http://doi.org/10.15359/ree.25-3.1

http://www.una.ac.cr/educare

educare@una.ac.cr

\section{Conclusiones}

En una primera instancia, se infiere de esta experiencia interuniversitaria, como se corrobora con otros estudios (Moreno Martínez y Leiva Olivencia, 2017), el elevado potencial educativo que se percibe en la RA. Por otra parte, Han et al. (2015) manifiestan que es fácil de integrar en las programaciones curriculares, pues el estudiantado posee móviles y considera que dicha tecnología es útil para su futuro profesional (Barroso Osuna y Gallego Pérez, 2017; Blas Padilla et al., 2019; Cabero Almenara et al., 2020). Asimismo, ellas favorecen la construcción de competencias emergentes sobre utilización de TIC, capacidad de trabajo en equipo y la dinamización de nuevos objetos curriculares inmersivos útiles para enriquecer los procesos de aprendizaje y nuevos recursos para el futuro profesional de la educación. En última instancia, se estima que es necesario utilizar objetos de aprendizaje aumentados en los ecosistemas curriculares para favorecer la competencia digital del estudiantado y la innovación sostenible educativa.

\section{Agradecimientos}

El presente trabajo se inserta en el proyecto de investigación denominado Realidad aumentada para aumentar la formación. Diseño, producción y evaluación de programas de realidad aumentada para la formación universitaria (RAFODIUN) (EDU2014-57446-P). También, se agradece al estudiantado de las UPO y UCSG, por su participación en la puesta en práctica de la experiencia innovadora inmersiva.

\section{Declaración de Material complementario}

Este artículo tiene disponible, como material complementario:

-La versión preprint del artículo en https://doi.org/10.5281/zenodo.4795905

\section{Referencias}

Azuma, R. T. (1997). A survey of augmented reality. En T. Ronald Azuma. Presence: Teleoperators and virtual environments (pp. 6355-6385). MIT Press.

Barroso Osuna, J. M. y Gallego Pérez, Ó. M. (2017). Producción de recursos de aprendizaje apoyados en realidad aumentada por parte de estudiantes de magisterio. Revista de Educación Mediática y TIC, 6(1), 23-38. https://doi.org/10.21071/edmetic.v6i1.5806

Billinghurst, M. y Dunser, A. (2012). Augmented reality in the classroom. Computer, 45(7), 56-63. 10.1109/MC.2012.111

Blas Padilla, D., Vázquez-Cano, E., Morales Cevallos, M. B. y López Meneses, E. (2019). Uso de apps de realidad aumentada en las aulas universitarias. Campus Virtuales, 8(1), 37-48. http:// uajournals.com/ojs/index.php/campusvirtuales/article/view/379 
http://doi.org/10.15359/ree.25-3.1

http://www.una.ac.cr/educare educare@una.ac.cr

Bressler, D. M. y Bodzin, A. M. (2013). A mixed methods assessment of students' flow experiences during a mobile augmented reality science game. Journal of Computer Assisted Learning, 29(6), 505-517. https://doi.org/10.1111/jcal.12008

Brinson, J. R. (2017). A further characterization of empirical research related to learning outcome achievement in remote and virtual science labs. Journal of Science Education and Technology, 26(5), 546-560. https://doi.org/10.1007/s10956-017-9699-8

Cabero-Almenara, J., Vázquez-Cano, E. y López-Meneses, E. (2018). Uso de la realidad aumentada como recurso didáctico en la enseñanza universitaria. Formación Universitaria, 11(1), 25 34. https://doi.org/10.4067/S0718-50062018000100004

Cabero Almenara, J., Vázquez-Cano, E., López Meneses, E. y Jaén Martínez, A. (2020). Posibilidades formativas de la tecnología aumentada. Un estudio diacrónico en escenarios universitarios. Revista Complutense de Educación, 31(2), 143-154. https://doi.org/10.5209/rced.61934

Caldwell, J. E. (2007). Clickers in the large classroom: Current research and best-practice tips. Cell Biology Education, 6(1), 9-20. https://doi.org/10.1187/cbe.06-12-0205

Carretero, S., Vuorikari, R. y Punie, Y. (2017). DigComp 2.1: The digital competence framework for citizens. With eight proficiency levels and examples of use. European Union. https://doi. org/10.2760/38842

Chang, H.-Y. (2018). Students' representational competence with drawing technology across two domains of science. Science Education, 102(5), 1129-1149. https://doi.org/10.1002/sce.21457

Chang, H.-Y., Wu, H.-K. y Hsu, Y.-S. (2013). Integrating a mobile augmented reality activity to contextualize student learning of a socioscientific issue. British Journal of Educational Technology, 44(3), E95-E99. https://doi.org/10.1111/j.1467-8535.2012.01379.x

Chang, S.-C. y Hwang, G.-J. (2018). Impacts of an augmented reality-based flipped learning guiding approach on students' scientific project performance and perceptions. Computers \& Education, 125, 226-239. https://doi.org/10.1016/j.compedu.2018.06.007

Chao, J., Chiu, J. L., DeJaegher, C. J. y Pan, E. A. (2016). Sensor-augmented virtual labs: Using physical interactions with science simulations to promote understanding of gas behavior. Journal of Science Education and Technology, 25(1), 16-33. https://doi.org/0.1007/s10956-015-9574-4

Chiang, T. H. C., Yang, S. J. H. y Hwang, G.-J. (2014). An augmented reality-based mobile learning system to improve students' learning achievements and motivations in natural science inquiry activities. Educational Technology y Society, 17(4), 352-365. http://www.jstor.org/ stable/jeductechsoci.17.4.352 
http://doi.org/10.15359/ree.25-3.1

http://www.una.ac.cr/educare

educare@una.ac.cr

Cózar Gutiérrez, R., de Moya Martínez, M. D. V., Hernández Bravo, J. A. y Hernández Bravo, J. R. (2015).Tecnologías emergentes para la enseñanza de las ciencias sociales. Una experiencia con el uso de realidad aumentada en la formación inicial de maestros. Digital Education Review, (27), 138-153. https://doi.org/10.1344/der.2015.27.138-153

Fernández Robles, B. (2018). La utilización de objetos de aprendizaje de realidad aumentada en la enseñanza universitaria de educación primaria. International Journal of Educational Research and Innovation, (9), 90-104. https://www.upo.es/revistas/index.php/IJERI/article/ view/2599

Fombona Cadavieco, J. y Vázquez-Cano, E. (2017). Posibilidades de utilización de la geolocalización y realidad aumentada en el ámbito educativo. Educación XX1, 20(2), 319342. https://doi.org/10.5944/educxx1.19046

Froehlich, D. E. 2018). Non-technological learning environments in a technological world: Flipping comes to the aid. Journal of new Approaches in Educational Research, 7(2), 88-92. https://doi.org/10.7821/naer.2018.7.304

Han, J. H., Jo, M., Hyun, E. y So, H.-J. (2015). Examining young children's perception toward augmented reali- ty-infused dramatic play. Education Technology Research Development, 63(3), 455-474. https://doi.org/10.1007/s11423-015-9374-9

Hood Cattaneo, K. (2017). Telling active learning pedagogies apart: From theory to practice. Journal of New Approaches in Educational Research, 6(2), 144-152. https://doi.org/10.7821/ naer.2017.7.237

Hunsu, N. J., Adesope, O. y Bayly, D. J. (2016). A meta-analysis of the effects of audience response systems (clicker-based technologies) on cognition and affect. Computers \& Education, 94, 102-119. https://doi.org/10.1016/J.COMPEDU.2015.11.013

Instituto Nacional de Tecnologías Educativas y de Formación del Profesorado. (2017). Marco común de competencia digital docente. http:// educalab.es/documents/10180/12809/ MarcoComunCompeDigiDoceV2.pdf

Jiménez Hernández, D., Sancho Requena, P. y Sánchez Fuentes, S. (2019). Perfil del futuro docente: Nuevos retos en el marco de EEES. Contextos Educativos. Revista de Educación, (23), 125-139. https://doi.org/10.18172/con.3471

Kim. K., Hwang, J.y Zo, H. (2016). Understanding users' continuance intention toward smartphone augmented reality applications. Information Development, 32(2), 161-174. https://doi. org/10.1177/0266666914535119 
http://doi.org/10.15359/ree.25-3.1

López Belmonte, J., Pozo Sánchez, S., Morales Cevallos, M. B. y López Meneses, E. (2019). Competencia digital de futuros docentes para efectuar un proceso de enseñanza y aprendizaje mediante realidad virtual. EDUTEC. Revista Electrónica de Tecnología Educativa, 67, 1-15. https://doi.org/10.21556/edutec.2019.67.1327

Moreno Martínez, N. N. y Leiva Olivencia, J. J. (2017). Experiencias formativas de uso didáctico de la realidad aumentada con alumnado del grado de educación primaria en la universidad de Málaga. Revista de Educación Mediática y TIC, 6(1), 81-104. https://doi.org/10.21071/ edmetic.v6i1.5809

Pedraza Goyeneche, C. E., Amado Plata, O. F., Lasso Cárdenas, E. y Munévar García, P. A. (2017). La experiencia de la realidad aumentada (RA) en la formación del profesorado en la Universidad Nacional Abierta y a Distancia UNAD Colombia. Pixel-Bit. Revista de Medios y Educación, (51), 111-131. https://doi.org/10.12795/pixelbit.2017.i51.08

Pool-Cibrian, W. J. y Martínez-Guerrero, J. I. (2013). Autoeficacia y uso de estrategias para el aprendizaje autorregulado en estudiantes universitarios. Revista Electrónica de Investigación Educativa, 15(3), 21-37. http://www.scielo.org.mx/scielo.php?script=sci arttext\&pid=S1607-40412013000300002\&lng=es\&tlng=es.

Rodríguez-García, A. M., Cáceres Reche, M. P. y Alonso García, S. (2018). La competencia digital del futuro docente: Análisis bibliométrico de la productividad científica indexada en Scopus. International Journal of Educational Research and Innovation, (10), 317-333. https:// www.upo.es/revistas/index.php/IJERI/article/view/2960

Santos, M. E. C., Wolde Lüke, A. I., Taketomi, T., Yamamoto, G., Rodrigo, M. M. T., Sandor, C. y Kato, H. (2016). Augmented reality as multimedia: The case for situated vocabulary learning. Research and Practice in Technology Enhanced Learning, 11(1), 1-23. https://doi.org/10.1186/ s41039-016-0028-2.

Vázquez Cano, E. (2021). Medios, recursos didácticos y tecnología educativa. UNED.

Wojciechowski, R. y Cellary, W. (2013). Evaluation of learners' attitude toward learning in ARIES augmented reality environments. Computers \& Education, 68, 570-585. https://doi. org/10.1016/j.compedu.2013.02.014 